\title{
Designing a Self-Efficacy Game for Health Literacy in Marginalized Communities
}

\author{
Morgan C. Evans \\ Carnegie Mellon University \\ Pittsburgh, USA \\ morganev@cs.cmu.edu \\ Junchao Lin \\ Carnegie Mellon University \\ Pittsburgh, USA \\ junchaol@andrew.cmu.edu
}

\author{
Adela Kapuścińska \\ Carnegie Mellon University \\ Pittsburgh, USA \\ kapuscinska.adela@gmail.com \\ Xuanyuan Liu \\ Carnegie Mellon University \\ Pittsburgh, USA \\ lxuanyua@andrew.cmu.edu
}

\author{
Maya Greenholt \\ Carnegie Mellon University \\ Pittsburgh, USA \\ mgreenho@andrew.cmu.edu \\ Tianyi Zhang \\ Carnegie Mellon University \\ Pittsburgh, USA \\ tz3@andrew.cmu.edu
}

\author{
Jessica Hammer \\ Carnegie Mellon University \\ Pittsburgh, USA \\ hammerj@andrew.cmu.edu
}

\author{
Geoff Kaufman \\ Carnegie Mellon University \\ Pittsburgh, USA \\ gfk@andrew.cmu.edu
}

\begin{abstract}
Health inequity is a critical problem in the United States and one that primarily affects marginalized communities. One critical aspect to interventions addressing this issue is the aim to increase health literacy, so that members of these communities can make informed decisions and feel empowered to take charge of their personal health. Our team developed a transformational game that uses self-efficacy to address players' health literacy in context. Using properties from both visual novel and strategy simulation genres, we present a game in which players take on the role of a community manager who works to better their community by completing quests from non-player character (NPC) community members. The current paper contributes our research and iterative design processes, and highlights future directions utilizing focus groups and playtesting with community members, game development, and evaluation studies to assess impact.
\end{abstract}

\section{CCS CONCEPTS}

- Applied computing $\rightarrow$ Interactive learning environments; - Human-centered computing $\rightarrow \mathrm{HCI}$ theory, concepts and models.

\section{ACM Reference Format:}

Morgan C. Evans, Adela Kapuścińska, Maya Greenholt, Junchao Lin, Xuanyuan Liu, Tianyi Zhang, Jessica Hammer, and Geoff Kaufman. 2021. Designing a Self-Efficacy Game for Health Literacy in Marginalized Communities. In CHI Conference on Human Factors in Computing Systems Extended Abstracts (CHI '21 Extended Abstracts), May 8-13, 2021, Yokohama, Japan. ACM, New York, NY, USA, 6 pages. https://doi.org/10.1145/3411763.3451609

This work is licensed under a Creative Commons

Attribution-NonCommercial-NoDerivs International 4.0 License.

CHI '21 Extended Abstracts, May 8-13, 2021, Yokohama, Japan

(c) 2021 Copyright held by the owner/author(s).

ACM ISBN 978-1-4503-8095-9/21/05.

https://doi.org/10.1145/3411763.3451609

\section{INTRODUCTION}

The need to address health inequities, defined as "systematic, potentially avoidable differences in health-or in the major socially determined influences on health-between groups of people who have different relative positions in social hierarchies according to wealth, power, or prestige" [7] has been identified as an issue of the utmost urgency on the global agenda [8]. Increasing health equity requires interventions to mitigate the social, economic, and environmental disadvantages experienced in marginalized communities, including racial and ethnic minority groups; people who have low income or low educational attainment; and rural residents [1]. Factors such as barriers in access to care, persistent and pervasive racial and ethnic biases in healthcare delivery, and lower levels of trust in healthcare systems exhibited by members of vulnerable groups have combined to exacerbate disparities in health literacy and outcomes.

To illustrate: the 2016 National Healthcare Quality and Disparities Report found that Hispanics in the United States experienced worse access to care compared with Caucasian patients for $75 \%$ of the measures included in the study, such as effective treatment and care coordination [36]. The COVID-19 pandemic has put health disparities faced by racial/ethnic minority groups in stark relief. Early reports have indicated that nearly $30 \%$ of COVID-19 cases have occurred in Black Americans, who constitute only $13 \%$ of the U.S. population [39]. At the same time, a recent survey found that only $42 \%$ of Black Americans intend to receive the COVID-19 vaccine as compared to $61 \%$ of White adults, which the researchers attributed to Black Americans' justifiable mistrust in inequitable healthcare systems [21].

Addressing health inequities among marginalized populations is a complex, multi-layered challenge that requires intervening at every level of the healthcare ecosystem. One important aspect, the one that guides the present work, involves creating interventions that increase both health literacy, defined as the "degree to which individuals have the capacity to obtain, process and understand basic health information and services needed to make appropriate 
health decisions" [35], and health self-efficacy, the belief in one's ability to act to produce desired results [4]. The combination of improved knowledge and self-belief in one's ability to access vital community resources is particularly important for members of marginalized communities to make informed decisions and take charge of their personal health.

Building on prior research showing the potential of simulation games and interactive narratives to model effective decision-making [6] and, in the context of health, to improve players' skill development and rehearsal [5], health knowledge [34], and self-confidence [29], the present work focuses on designing a novel digital game intervention for promoting health literacy and self-efficacy among members of marginalized populations. Specifically, we propose a digital game design in which players are placed in the role of community manager, with the goal of connecting community members to vital individuals, institutions, and resources, as a means to provide players with transferable skills in accessing, understanding and utilizing relevant health resources in their own context outside of the game. We present the foundational literature that informed our specific game design goals, discuss the methods we used to define the focus and format for the game, present a description of the in-progress game prototype we are developing, and look ahead to the next steps in our game design and evaluation process.

\section{LITERATURE REVIEW}

Our literature review focused on health literacy in marginalized communities as well as game genres that would be most conducive for achieving our transformative goals related to health literacy and self-efficacy.

\subsection{Health literacy in marginalized communities}

In recent years, $\mathrm{HCI}$ research has seen an increasing focus on understanding the experiences and needs of under-served social groups and designing technologies that aim to support and empower members of marginalized populations ([14], [16], [37]). This growing body of work includes research addressing the focal goals of increasing health literacy and health self-efficacy that guide the present work. For example, Saksono et al.[37] studied low socio-economic status (SES) families' perceptions of and access to wearable technologies that track physical activity and have shown that designing those technologies to support families in interpreting their own activity data can support more effective experiential learning [38]. Lakerveld et al. [32] proposed a cardio-metabolic health intervention for low-SES groups that incorporated context sensing and situated "nudges" for improving food purchasing decisions and prompting physical activity in appropriate times and settings. Devito et al. [13] have pointed out the need for social technologies to account for the specific health literacy challenges faced by members of marginalized groups, including known issues with disinformation and misinformation that those groups must confront in accessing digital health resources (e.g., information on safer sex practices among LGBTQ+ or low-SES individuals). These examples illustrate the importance of carefully considering the social and physical contexts in which health information is accessed and used, as well as the need to provide forms of support for promoting health literacy that are tailored to the unique needs and challenges of specific marginalized groups.

To this end, HCI researchers have developed new design methods and frameworks for designing for and with - marginalized groups. For example, Harrington and colleagues [25, 26] have developed participatory design methods, including community-based design workshops, that center the voices of members of under-served groups (e.g., low-SES, older African Americans) in surfacing health challenges and envisioning new technologies to address them. Erete et al. [17] stress the importance of taking an intersectional approach in designing for marginalized groups: how axes of domination, privilege, and oppression interact to affect the needs and experiences of specific groups in distinct ways.

We take direct inspiration from these bodies of work. In our design approach, we carefully consider the social environments of our target participants, their social bonds and networks (such as connections with caregivers and local resources), and key facets of their social identity, including race, gender, and socioeconomic status. In addition, as detailed in our future work, we will use research through design methods [42] to center the perspectives and insights of our target groups as co-creators.

\subsection{Game-based Interventions for Promoting Health Literacy and Self-Efficacy}

A growing body of work has focused on the design of evaluation of serious games developed as health interventions, with self-efficacy theory being one of the most influential forces guiding their design [33]. As defined by Bandura, self-efficacy refers to individuals' belief in their own ability to accomplish a certain task or goal [2]. Later Bandura proposed building self-efficacy for changing self-directed behaviors, including in the domain of personal health, requires providing opportunities to: 1) access, interpret, and apply information about a particular behavior (including information about risks and benefits); 2) the opportunity to learn and develop skills; 3) guided practice in acquiring and exhibiting those skills and 4) a strong network of support for providing emotional and instrumental support [3].

In a critical analysis of games for health, Lieberman [33], argues that games offer an ideal venue for acquiring new knowledge and skills by affording players the ability to learn and repeat new healthrelated behaviors, to experience guidance and support through rolemodel characters, and to play out decisions and experience their consequences within the confines of the game. Moreover, prior work on narratives and games have revealed their value for instilling new knowledge, attitudes, and behaviors in ways that mitigate the potential psychological threat of confronting self-relevant information (such as gaps in knowledge or skills). For example, highly transporting stories and games have been shown to make readers and players more receptive to new information and perspectives [22], and deep connections with the characters one embodies in games can promote the internalization of beliefs and behaviors that persist outside of the game [31]. The psychological distancing afforded by narrative- and character-driven games is particularly important for engaging players with sensitive topics [30] and, moreover, aligns with recommendations for designing culturally sensitive health interventions for (and with) marginalized populations [15, 19, 28]. 
This prior work would suggest that game genres that are both character-driven and offer players ample opportunities to make decisions about characters' health behaviors and outcomes are ideal for building health literacy and self-efficacy. Two such genres that have been the focus of several successful serious games are visual novels and strategy simulation games. Visual novels are interactive narrative games that feature branching storylines determined by players' choices at key decision points [9]. As such, visual novels are highly conducive to modeling cause-and-effect relationships and providing a sense of agency and autonomy to players to determine the trajectory of the story [12, 24]. In a similar vein, strategy simulation games (or strategy sims for short) aim to model real-life situations by putting players directly into contexts in which they simulate judgment and decision-making processes; prior work has revealed their value in instilling disciplinary knowledge [20] and building domain-specific self-efficacy [41].

We took inspiration from a number of games that successfully incorporated elements from visual novels and strategy sims in determining the direction for our own game design. First and foremost, Elm City Stories, is a visual novel designed to provide at-risk adolescents from marginalized groups (e.g., racial minorities, sexual or gender minorities, low-SES populations) the opportunity to acquire and practice skills intended to sexual risk behaviors, with the ultimate goal of preventing HIV transmission [27]. In the game, players create aspirational avatars, intended to represent their own future hopes and dreams, and travel from 7 th to 12 th grade, confronting challenges and making decisions relevant to sexual and relationship health. A clinical randomized trial revealed that the game had a beneficial effect on youth players' sexual knowledge and attitudes toward sexual health [18]. OrderUP is a mobile health game in which players learn how to make healthier meal choices; the game applies skill development and guided practice, key elements of the self-efficacy framework, to improve players' understanding of how to eat healthfully, engage in nutrition-related analytical thinking, and reevaluate the healthiness of their real-life habits [23]. Finally, Hacked Time is a desktop game that aims to instill improved cybersecurity behaviors in players by putting them in the role of a time-traveling detective who advises college students about cybersecurity risks and provides guided practice in improving skills such as creating secure account passwords and utilizing two-factor authentication. A randomized control trial showed that the game was effective at increasing players' cybersecurity knowledge and self-efficacy [10].

\section{RESEARCH AND DESIGN METHODS}

We describe our process using Tandem Transformational Design [40], which is a framework conducive for our interdisciplinary team of researchers and game designers. This framework hinges on first delineating a transformational goal, informed by prior theory and empirical findings, which is then used as the shared vision for goaldriven game design, in tandem [40]. In the following subsections we describe our transformative goals, as derived from self-efficacy theory; describe our game concept generation process; and, finally, present a detailed description of the game concept that emerged from the team's process of aligning goals with game mechanics.

\subsection{Literature Review Process}

Prior to initiating any design work, we mapped our knowledge through the Transformational Game Design Framework [11] to define the high level purpose of our game. Through this process, we identified our target demographic as members of marginalized communities, transformative goals towards promoting health literacy and self-efficacy (wanted transformations), as well as general barriers that may obstruct the player from achieving one or many of the wanted transformations. Table 1 gives an example of the aforementioned. Natural dependencies occur between the two columns, for example, we want our players to feel empowered to engage with their personal health by being comfortable asking the doctor questions relevant to their personal health (Self-advocacy). A negative experience with previous healthcare may lessen their incentive to engage (Motivation).

\subsection{Generation of games}

The following constraints were motivated by our literature review and Transformational Framework, and were determined in the Alignment stage of Tandem Transformational Design Framework: types of users (single player, family-mediated player, libraryfacilitated play), artifacts involved (online portal/database; journal; power of family/community) and self-efficacy concepts (skill development, guided practice, and social support). During a series of design rounds, three designers generated game design sketches, within constraints provided by the lead game designer. The designers analyzed their generated sketches in terms of common themes (e.g., simulation, time travel, emphasis on family) and literature concepts (e.g., self-efficacy) until the team decided sufficient coverage on the guiding themes and goals from the literature was achieved. A representative sample of the 16 game design sketches' strengths are presented in Figure 1.

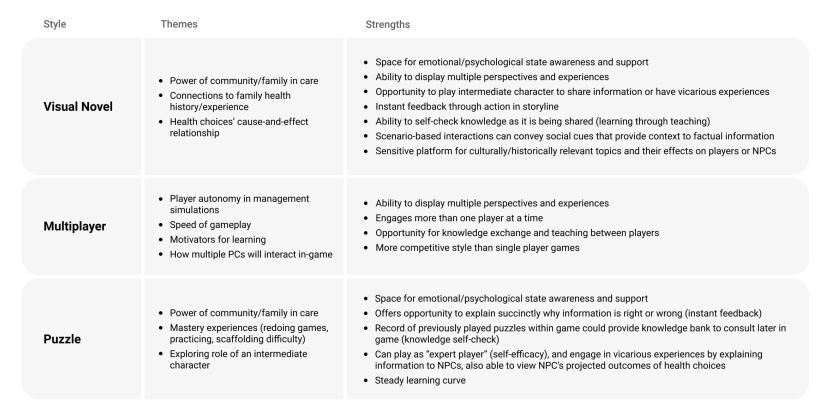

Figure 1: Strengths of Generated Game Design Sketches

Next, the lead game designer synthesized the 16 game design sketches vis-à-vis the research and design implications. In the synthesis, she identified their themes, core concepts, factors, paradigms, game references and game character types. One of the artifacts of this process was Figure 1, where she mapped out each game design sketch by its leading self-efficacy concepts, thus highlighting the designers' conceptual leanings towards concrete solutions to the design brief. However, her primary task was to bring out their strong and useful elements and cluster those that would function 
Table 1: Transformational Framework Findings with Examples

\begin{tabular}{ll}
\hline \multicolumn{1}{c}{ Wanted Transformations } & \multicolumn{1}{c}{ Anticipated Barriers } \\
\hline Behavior: create routines for health check-ins & Access: lack of access to technology or factual information \\
Self-advocacy: comfortable asking questions & Motivation: lack of incentive possibly due to a negative experience \\
Knowledge: know where to find resources & Relevance: lack of cultural representation \\
Skill: control of personal health & Unfamiliarity: lack of awareness of available health resources \\
\hline
\end{tabular}

best together, as a reflection on effective mechanical, narrative and literature combinations. For example, by designing the game's main character (the community manager) as a non-expert in health literacy but a figure of authority motivated to increase their knowledge (to better support their community), we present players with a learning trajectory into mastery. This process resulted in three high-level design clusters that addressed the design brief in different ways. Next, the project leadership held a workshop with these three design clusters using the 1-minute of gameplay brainstorming method, writing out what a 1-minute of the gameplay loop could look like, generating nine sketches.

\subsection{Evaluation of concepts}

To choose the final design, the project leadership deliberated and identified the designs they thought held the most promise. Literature and research considerations were key in brainstorming, iterating, and analyzing designs throughout the goal-driven game design process. In the "winning" game concept, the player takes on the role of a community manager where they manage interpersonal relationships in the community by helping NPCs solve personal problems. Throughout the game, the player encounters a variety of topics related to the narratives of those members in the community, largely including health literacy. The player character is embedded in the community and a non-expert in health literacy. We believe this positions them as a key player in the NPCs' interventions, all while motivating the player to foster the skills to solve the problem, practice by making decisions, and maintaining social support.

\section{GAME PROPOSAL}

Our game uses a combination of elements from visual novels and strategy simulation games. Most of the game's action occurs within the map overview of the community in which they reside. Here, the User Interface informs players of their progress via city stats. In map view, the player is able to click on institutions in the city (such as the library) to gain knowledge about its role in the city including available resources. The player is also able to interact with NPCs on the map to learn of what problems ail them by clicking on their location to enter the dialogue view. As in visual novel games, the player is encouraged to click through and read the narrative, while making dialogue choices for the community manager. Their decisions determine what information gets revealed. For example, Sophia Vargas might disclose to the community manager that she is looking for a part-time job to surprise her family. Over many conversations with community members, the player is able to build out their Journal/Info Bank with information on the goings-on in the community. The more information they gather, the better, as it can be relevant to the solution of other NPCs' quests.
Throughout the game, notifications will appear on the map to request the community manager's help with solving a problem (Quest) for each member in the community. NPCs each have five quests: two related to their core narrative arc (e.g. Sophia Vargas wants to improve how she supports her family before she goes to college), two related to health literacy (e.g. Sophia Vargas wants to learn about and document her family's health history), and one related to their relationship with the player (e.g. Sophia Vargas invites the community manager to her basketball match). By completing quests for NPCs, the player increases community engagement. The game is won when the player gets $100 \%$ community engagement. Figure 2 illustrates the Core Loop.

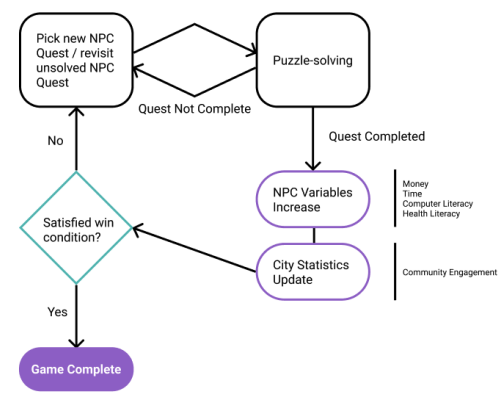

Figure 2: Core Loop

Quests are solved by connecting a piece of information present in the player's Journal/InfoBank with the active quest: they simply bring it up with the NPC. For example, the player might connect Sophia Vargas to Ms. Sylvia, who was looking for somebody to teach her how to use the computer, to resolve both of their quests. When the player connects information with a quest, they receive feedback from the NPC with the outcome of that suggestion. If the player is in a collaborative setting, they are prompted to discuss the decision amongst the group prior to receiving feedback from the NPC. When completing an NPC's quest, community engagement points are awarded based on viable solutions. Conversely, we don't introduce de facto wrong choices: red herring choices may be presented to the player to test their assessment and help them reflect on why some choices could've been more effective than others.

\subsection{Self-efficacy and health literacy}

We now describe how our game design embodies self-efficacy and health literacy using Bandura's framework [3].

Risk information The player learns most directly about health literacy concepts in the visual novel mode of our game, where they gain exposure to health concepts from NPCs. This information 
is exposed to the player through a diversity of NPC narratives, presented either as a description of an NPCs quest (e.g. Ms. Silva needs help filling her prescription because she has diabetes) or presented in the Journal/InfoBank as information automatically extracted from an NPC's narrative (e.g. people with diabetes need to take insulin). Once the quest is accepted, the player engages with the information they learned by checking the Journal/Info bank and assess the risk of using information they already have to solve the quest, or wait until they discover more possible solutions.

Skill development To win the game, the player must achieve $100 \%$ community engagement by completing all of the NPCs quests. To that end, players must engage with community members in order to learn information about their narrative including unlocking quests (e.g., you learn Sophia needs a job and are given the option to activate the quest) as well as factual information (e.g., you learn the generic name for Tylenol which is added to your InfoBank). The player makes dialogue choices to determine the information revealed by the NPC. The decision to use specific factual information to solve a quest is another opportunity to learn skills related to any given quest, whether the quest is related to health literacy, the NPC's core narrative, or the player's relationship to the NPC.

Guided practice For all decisions the player makes in our game, we will implement appropriate feedback to help the player understand the result of their actions. Most notably, once the player offers a solution to the NPC for an active quest, the player receives feedback from them describing how the player's solution ended up helping them. In practice, we will present this feedback after the player is offered time to consider the effects of the solution, or a discussion may be prompted at this time in the event they are playing in a shared environment, perhaps with family. The player will still receive feedback in the community engagement city stat, even if the solution they offered the NPC was not the most optimal. Finally, the player receives feedback on how they have navigated the conversation around the NPC's narrative by how the conversation progresses and the information that is revealed by the NPC.

Social support As the community manager, the player is the embodiment of a community advocate for health literacy as well as interpersonal connections. Completing a quest requires the player to progressively learn and understand multiple characters' narratives; it is only through conversation and connection that the player is able to gain knowledge and collect information that is a possible solution to active quests. Lastly, the player receives social support through encouraging feedback from NPCs upon the completion of each of their quests. This feedback will detail the positive attributes of the players solution even if it was not the optimal solution for that quest.

\subsection{Marginalized communities}

In order to better serve marginalized populations, our design aims to create a game narrative and context that will resonate with the lived experiences of members of these communities. We are designing a diverse group of NPCs in the game community, who belong to various marginalized groups; they could be people of color, people with low socioeconomic status (SES), elders, immigrants, people with disabilities, people in the LGBTQ+ community. Designing interpersonal relations, we would also break the nuclear family stereotypes (i.e. a family that consists of two parents and their children) to reflect more complex and realistic family and community structures. Secondly, as we are designing a digital game, we plan to accommodate different levels of technology experience and accessibility. For players with little prior game experience, we will incorporate simple game mechanisms, such as a point-and-click interface, to make the game easier to learn, more approachable, and paced by the readers' preferred reading speed.

\section{CONCLUSION AND FUTURE WORK}

In this paper, we have described our research and design processes towards our design of a transformational game that increases health literacy and self-efficacy for marginalized communities. Our proposed game design addresses skill development through decisionmaking, mapping health information flow, and giving recommendations to community members for best practices. Through our use of visual novel, the player is immersed into the narrative contexts of a diversity of community member experiences. To win the game, the player accrues social engagement points by way of engaging with NPCs by providing social support; the player also receives support in the feedback provided by NPCs with the effects of the players suggestion.

In future work, we will conduct focus groups with members of these communities to better address their needs and experiences, and ensure that our game is culturally sensitive. We hope that through these focus groups we can inform our narratives that addresses relatable pain points or concerns, such as limited resources and distrust in healthcare systems. Through completing quests, the player as a community manager would help build deeper connections with and in between other NPCs in the community, feeling a sense of belonging and empowerment, which we hope to translate to their real-life experience as confidence and motivations. We note that at the time of writing this paper, one limitation to our current design is the limited involvement of both players and health experts. We will develop the prototype of the game based on our pre-production work and iterate the design towards various modes of play, including single player and family-mediated play and based on playtesting with relevant stakeholders. Finally, we will use a randomized control trial to quantitatively evaluate the effect of our community manager game on individual's health literacy.

\section{ACKNOWLEDGMENTS}

Funded under cooperative agreement number UG4LM012345 with the University of North Texas Health Science Center - Gibson D. Lewis Library, and awarded by the DHHS, NIH, National Library of Medicine.

\section{REFERENCES}

[1] Foster Osei Baah, Anne M Teitelman, and Barbara Riegel. 2019. Marginalization: Conceptualizing patient vulnerabilities in the framework of social determinants of health-An integrative review. Nursing inquiry 26, 1 (2019), e12268.

[2] Albert Bandura. 1977. Self-efficacy: toward a unifying theory of behavioral change. Psychological review 84, 2 (1977), 191.

[3] Albert Bandura. 1990. Perceived self-efficacy in the exercise of personal agency. Journal of applied sport psychology 2, 2 (1990), 128-163.

[4] Albert Bandura. 2010. Self-efficacy. The Corsini encyclopedia of psychology (2010), $1-3$. 
[5] Moderator: Tom Baranowski, Participants: Debra Lieberman, Richard Buday, Wei Peng, Lukas Zimmerli, Brenda Wiederhold, and Pamela M Kato. 2013. Videogame mechanics in games for health. GAMES FOR HEALTH: Research, Development, and Clinical Applications 2, 4 (2013), 194-204.

[6] Alexandre Bran and David Vaidis. 2019. Choose your own risks: measuring risk-taking through an interactive novel. (2019).

[7] Paula Braveman. 2006. Health disparities and health equity: concepts and measurement. Annu. Rev. Public Health 27 (2006), 167-194.

[8] Ali Murad Büyüm, Cordelia Kenney, Andrea Koris, Laura Mkumba, and Yadurshini Raveendran. 2020. Decolonising global health: if not now, when? BMF Global Health 5, 8 (2020), e003394.

[9] Dani Cavallaro. 2009. Anime and the visual novel: narrative structure, design and play at the crossroads of animation and computer games. McFarland.

[10] Tianying Chen, Margot Stewart, Zhiyu Bai, Eileen Chen, Laura Dabbish, and Jessica Hammer. 2020. Hacked Time: Design and Evaluation of a Self-Efficacy Based Cybersecurity Game. In Proceedings of the 2020 ACM Designing Interactive Systems Conference. 1737-1749.

[11] Sabrina Culyba. 2018. The Transformational Framework: A process tool for the development of Transformational games. Carnegie Mellon University.

[12] Timothy Day and Jichen Zhu. 2017. Agency informing techniques: Communicating player agency in interactive narratives. In Proceedings of the 12th International Conference on the Foundations of Digital Games. 1-4.

[13] Michael A Devito, Ashley Marie Walker, Jeremy Birnholtz, Kathryn Ringland, Kathryn Macapagal, Ashley Kraus, Sean Munson, Calvin Liang, and Herman Saksono. 2019. Social Technologies for Digital Wellbeing Among Marginalized Communities. In Conference Companion Publication of the 2019 on Computer Supported Cooperative Work and Social Computing. 449-454.

[14] Tawanna R Dillahunt. 2014. Fostering social capital in economically distressed communities. In Proceedings of the SIGCHI Conference on Human Factors in Computing Systems. 531-540.

[15] Mohan J Dutta. 2007. Communicating about culture and health: Theorizing culture-centered and cultural sensitivity approaches. Communication Theory 17 , 3 (2007), 304-328.

[16] Sheena Erete and Jennifer O Burrell. 2017. Empowered participation: How citizens use technology in local governance. In Proceedings of the 2017 CHI Conference on Human Factors in Computing Systems. 2307-2319.

[17] Sheena Erete, Aarti Israni, and Tawanna Dillahunt. 2018. An intersectional approach to designing in the margins. Interactions 25, 3 (2018), 66-69.

[18] Lynn E Fiellin, Tassos C Kyriakides, Kimberly D Hieftje, Tyra M Pendergrass, Lindsay R Duncan, James D Dziura, Benjamin G Sawyer, and David A Fiellin. 2016. The design and implementation of a randomized controlled trial of a risk reduction and human immunodeficiency virus prevention videogame intervention in minority adolescents: PlayForward: Elm City Stories. Clinical Trials 13, 4 (2016), 400-408

[19] Leigh Arden Ford and Gust A Yep. 2003. Working along the margins: Developing community-based strategies for communicating about health with marginalized groups. Handbook of health communication (2003), 241-261.

[20] Aroutis N Foster. 2011. The process of learning in a simulation strategy game Disciplinary knowledge construction. Fournal of Educational Computing Research 45, 1 (2011), 1-27.

[21] Cary Funk and Alec Tyson. 2020. Intent to Get a COVID-19 Vaccine Rises to 60\% as Confidence in Research and Development Process Increases. Pew Research Center (December 2020) (2020).

[22] Melanie C Green and Timothy C Brock. 2000. The role of transportation in the persuasiveness of public narratives. Journal of personality and social psychology 79, 5 (2000), 701.

[23] Andrea Grimes, Vasudhara Kantroo, and Rebecca E Grinter. 2010. Let's play! Mobile health games for adults. In Proceedings of the 12th ACM international conference on Ubiquitous computing. 241-250.

[24] D Fox Harrell and Jichen Zhu. 2009. Agency Play: Dimensions of Agency for Interactive Narrative Design.. In AAAI spring symposium: Intelligent narrative technologies II. 44-52.

[25] Christina N Harrington. 2020. The forgotten margins: what is community-based participatory health design telling us? Interactions 27, 3 (2020), 24-29.

[26] Christina N Harrington, Lauren Wilcox, Kay Connelly, Wendy Rogers, and Jon Sanford. 2018. Designing Health and Fitness Apps with Older Adults: Examining the Value of Experience-Based Co-Design. In Proceedings of the 12th EAI International Conference on Pervasive Computing Technologies for Healthcare. 15-24.

[27] Kimberly Hieftje, Lynn E Fiellin, Tyra Pendergrass, and Lindsay R Duncan. 2016. Development of an HIV prevention videogame intervention: lessons learned. Int. 7. Serious Games 3, 2 (2016), 83-90.

[28] Sofia Hussain, Elizabeth B-N Sanders, and Martin Steinert. 2012. Participatory design with marginalized people in developing countries: Challenges and opportunities experienced in a field study in Cambodia. International fournal of Design 6, 2 (2012).

[29] Pamela M Kato, Steve W Cole, Andrew S Bradlyn, and Brad H Pollock. 2008. A video game improves behavioral outcomes in adolescents and young adults with cancer: a randomized trial. Pediatrics 122, 2 (2008), e305-e317.

[30] Geoff Kaufman and Mary Flanagan. 2015. A psychologically "embedded" approach to designing games for prosocial causes. Cyberpsychology: fournal of Psychosocial Research on Cyberspace 9, 3 (2015).

[31] Geoff F Kaufman and Lisa K Libby. 2012. Changing beliefs and behavior through experience-taking. Fournal of personality and social psychology 103, 1 (2012), 1

[32] Jeroen Lakerveld, Joreintje D Mackenbach, Femke De Boer, Boris Brandhorst, Jacqueline EW Broerse, Gert-Jan de Bruijn, Gerda Feunekes, Marleen Gillebaart, Marjolein Harbers, Jody Hoenink, et al. 2018. Improving cardiometabolic health through nudging dietary behaviours and physical activity in low SES adults: design of the Supreme Nudge project. BMC Public Health 18, 1 (2018), 899.

[33] Debra A Lieberman. 1997. Interactive video games for health promotion: Effects on knowledge, self-efficacy, social support, and health. Health promotion and interactive technology: Theoretical applications and future directions (1997), 103120

[34] Debra A Lieberman. 2006. What can we learn from playing interactive games? (2006)

[35] Lynn Nielsen-Bohlman, Allison M Panzer, David A Kindig, et al. 2004. The extent and associations of limited health literacy. In Health literacy: a prescription to end confusion. National Academies Press (US).

[36] US Department of Health, Human Services, et al. 2017. 2016 National Healthcare Quality and Disparities Report.

[37] Herman Saksono, Carmen Castaneda-Sceppa, Jessica Hoffman, Magy Seif El-Nasr, Vivien Morris, and Andrea G Parker. 2018. Family health promotion in low-SES neighborhoods: A two-month study of wearable activity tracking. In Proceedings of the 2018 CHI Conference on Human Factors in Computing Systems. 1-13.

[38] Herman Saksono, Carmen Castaneda-Sceppa, Jessica Hoffman, Magy Seif El-Nasr, Vivien Morris, and Andrea G Parker. 2019. Social reflections on fitness tracking data: A study with families in low-SES neighborhoods. In Proceedings of the 2019 chi conference on human factors in computing systems. 1-14.

[39] Neeta Thakur, Stephanie Lovinsky-Desir, Christian Bime, Juan P Wisnivesky, and Juan C Celedón. 2020. The Structural and Social Determinants of the Racial/Ethnic Disparities in the US COVID-19 Pandemic. What's Our Role? American journal of respiratory and critical care medicine 202, 7 (2020), 943-949.

[40] Alexandra To, Elaine Fath, Eda Zhang, Safnah Ali, Catherine Kildunne, Anny Fan, Jessica Hammer, and Geof Kaufman. 2016. Tandem Transformational Game Design: A Game Design Process Case Study. In Proceedings of the International Academic Conference on Meaningful Play.

[41] George H Tompson and Parshotam Dass. 2000. Improving students' self-efficacy in strategic management: The relative impact of cases and simulations. Simulation \& Gaming 31, 1 (2000), 22-41.

[42] John Zimmerman, Jodi Forlizzi, and Shelley Evenson. 2007. Research through design as a method for interaction design research in HCI. In Proceedings of the SIGCHI conference on Human factors in computing systems. 493-502. 